\title{
Effects of Low-Frequency Pulsed Electromagnetic Fields on High-Altitude Stress Ulcer Healing in Rats
}

\author{
Mingke Jiao, ${ }^{1,2}$ Hong Yin $\left(D,{ }^{1}\right.$ Jie Hu, ${ }^{3}$ Wenjuan Xu, ${ }^{2}$ Xiao Zhang, ${ }^{1}$ and Peng Zhang ${ }^{2}$ \\ ${ }^{1}$ The Departments of Radiology, Xijing Hospital, Fourth Military Medical University, Xi'an 710032, China \\ ${ }^{2}$ The Department of Medical Engineering, General Hospital of Xinjiang Military Region, Urumqi 830000, China \\ ${ }^{3}$ The Department of Echocardiography, Affiliated Traditional Chinese Medicine Hospital, Xinjiang Medical University, \\ Urumqi 830000, China
}

Correspondence should be addressed to Hong Yin; yinhong@fmmu.edu.cn

Received 27 March 2019; Accepted 26 May 2019; Published 11 June 2019

Academic Editor: Luenda Charles

Copyright (c) 2019 Mingke Jiao et al. This is an open access article distributed under the Creative Commons Attribution License, which permits unrestricted use, distribution, and reproduction in any medium, provided the original work is properly cited.

\begin{abstract}
High-altitude stress ulcer (HSU) has constantly been a formidable clinical challenge for high-altitude and severe hypoxia. Pulsed electromagnetic fields (PEMFs) have been verified to have the ability to penetrate tissues, and the biological effects have been confirmed effective on various tissue restorations. However, the therapeutic effect of PEMFs on HSU has been rarely reported. This study aimed to evaluate the effects of PEMFs on HSU healing systematically. Sprague-Dawley rats were assigned to control, HSU, and HSU+PEMF groups. The HSU models were induced by restraint stress under low-pressure hypoxia. The HSU+PEMF group was subjected to PEMF exposure. During the HSU healing, gastric juice $\mathrm{pH}$ values, ulcer index (UI), and histopathologic changes were investigated. Furthermore, tumor necrosis factor- $\alpha$ (TNF- $\alpha$ ) was determined to analyze the severity of gastric membrane inflammations. Norepinephrine (NE), which influences gastric acid secretion, was measured. Results indicated the UI of the HSU+PEMF decreased faster than that of the HSU group. Histopathologic observation suggested that the ulcer tissue healing is faster in the HSU+PEMF group than in the HSU group. The TNF- $\alpha$ /total protein results revealed that the inflammation of the HSU+PEMF group is suppressed effectively. The $\mathrm{pH}$ values are higher in the HSU+PEMF group than in the HSU, as confirmed by $\mathrm{NE}$ examination. The results indicated that low-frequency PEMFs can penetrate stomach tissues to relieve the symptoms of HSU and promote the regeneration of disturbed tissues, thus implying the clinical potential of PEMF therapy for HSU treatment.
\end{abstract}

\section{Introduction}

Stress ulcer mainly occurs in high-altitude and plateau regions due to severe hypoxia, colds, and other stresses [1]. An ulcer is accompanied by bleeding and infiltration of inflammatory cells into the gastric mucosa [2]. HSU in highaltitude and cold regions may result in heavy bleeding and perforation of the digestive tract, even death without timely and effectively treatment. As the main factors associated with the induction of HSU, cold and severe hypoxia increases the blood viscosity of gastric mucosa, thereby resulting in thrombosis and ulcer $[3,4]$. In addition, the environment stress, which induced the increased central nervous system activity, may increase gastric acid secretion and causes spasm on the small vessels of mucosa $[5,6]$. Currently, drugs and surgical resection remain the main therapy for gastric ulcers.
For example, certain drugs have been used to influence the gastric acid secretion and norepinephrine (NE). However, several side effects of drugs and surgery have caused injuries to patients, especially in high-altitude regions; these injuries include mental aberrations, asiderosis, and pneumonia [7-9]. Thus far, few methods have successfully treated HSU. Therefore, HSU remains a clinical challenge given complicated plateau stress caused by a combination of colds, hypoxia, and so on. Therefore, effective and low-cost therapies must be developed to accelerate HSU healing.

Substantial and growing evidence has confirmed that low-frequency pulsed electromagnetic fields (PEMFs), as an alternative noninvasive method, can efficiently induce tissue regeneration and cell proliferation [10-12]. Therefore, the positive effects of PEMFs on the healing of certain tissue damage have been investigated in numerous animal experiments 
$[13,14]$. In addition, clinical investigations have confirmed the efficacy of PEMFs in repairing impaired tissues $[4,15]$. Moreover, the penetration characteristic of PEMFs has been applied to treat injured tissues in deep layers [16]. Specifically, several experimental studies have demonstrated that PEMFs can adjust the NE level to minimize the psychological stresses of biology [1, 17]. HSU differs from other tissue injuries in many aspects. First, the cause of HSU is related to stresses [5], which may be associated with NE. Second, the injured tissues of HSU are found inside the body. Finally, HSU pathology is characterized by small vessel blockage and cell apoptosis. Therefore, to date, there have been no studies investigating the effectiveness of low-frequency PEMF exposure in accelerating HSU healing.

In the present study, we systematically determined the noninvasive low-frequency PEMFs on HSU in rats in terms of stress-induced gastric ulcer development or healing of tissue lesions. According to the positions of ulcer and previous studies, $15 \mathrm{~Hz}$ and $34 \pm 0.4 \mathrm{mT}$ of PEMFs were applied to effectively induce biological effects and tissue healing. Accordingly, NE was measured to determine the influence of PEMFs on central nervous system activity. In addition, gastric juice $\mathrm{pH}$ values, ulcer index (UI), associated protein of inflammation, and histological peculiarity of different groups were systematically determined and compared during HSU wound healing. Ultimately, the purpose of the research was to develop an appropriately characterized noninvasive treatment for HSU via low-frequency PEMFs.

\section{Materials and Methods}

2.1. Preparation of Animals. In this experiment, 72 SpragueDawley rats $(220 \pm 20 \mathrm{~g})$ of either sex were purchased from the Animal Center of the Fourth Military Medical University. The study was performed strictly in accordance with the guiding principles of the Institutional Animal Ethical Committee, the Committee for the Purpose of Control and Supervision of Experiments on Animals, and the Guide for the Care and Use of Laboratory Animals published by the National Institute of Health (NIH Publication No. 85-23). The animals received standard food and clean tap water ad libitum from an automated watering system and were kept at room temperature $\left(24 \pm 1^{\circ} \mathrm{C}\right)$ before HSU model construction.

2.2. HSU Models. The rats were assigned to three groups, namely, normal control $(\mathrm{n}=8)$, HSU $(\mathrm{n}=32)$, and HSU with low-frequency PEMF exposure (HSU+PEMF, $\mathrm{n}=32$ ). First, the rats from the HSU and HSU+PEMF groups were housed for $2 \mathrm{~h}$ a day in a large hypobaric chamber (DYC-3013M, Urumqi General Hospital of Lanzhou Military Region, Urumqi, China) with controlled temperature (24 \pm $\left.1^{\circ} \mathrm{C}\right)$, humidity $(50 \% \pm 10 \%)$, and pressure $(54.1 \pm 1.0 \mathrm{kPa})$ for 5 days to acclimatize them under hypoxic and lowpressure conditions. For the stomach to be involved in stress responses, during the acclimatization course, the rats were fasted, bounded, and immersed carefully in $10^{\circ} \mathrm{C}$ water with surface and breastbone line for half an hour per day. On the fifth day of the acclimatization, the rat stomachs were further perfused with $2 \mathrm{ml} / \mathrm{kg}$ pure alcohol to ensure a perfect
HSU model. After model establishment, the rats from the HSU+PEMF group were exposed to low-frequency PEMFs for $3 \mathrm{~h}$ daily at the normal comfortable environment $(24 \pm$ $\left.1^{\circ} \mathrm{C}\right)$ for 8 days. During the PEMF exposure, the rats were housed in transparent resin cages $(60 \mathrm{~mm} \times 120 \mathrm{~mm} \times 55 \mathrm{~mm})$ and diminished range of motion to ensure that the PEMF could focus on the stomach. During the HSU healing, all of the groups were individually housed and fed at a normal temperature $\left(24 \pm 1^{\circ} \mathrm{C}\right)$ and pressure $(95.3 \pm 1.3 \mathrm{kPa})$. At a 2 day interval during the HSU healing, the UI, juice $\mathrm{pH}$ values, histological changes, tumor necrosis factor- $\alpha$ (TNF- $\alpha$ )/total protein, and $\mathrm{NE}$ of eight rats from each group were examined and evaluated.

2.3. PEMF Exposures. During the ulcer healing, the rats from the HSU+PEMF group were subjected to a whole-body PEMF exposure for 7 days. The low-frequency PEMF facility (BPS-I, Urumqi, China; China Patent no. 201520046119.2) consisted of an electromagnetic generator and a transparent resin cage with focused coil (Figure 1(a)). The low-frequency pulsed signals were produced by the signal generator, which was designed with the chip-microcomputer (STC89C52, STC, Shenzhen, China). The frequency and duty cycle were adjusted and controlled with the specific program. And then the output signal amplitude was amplified by the power amplifier. The cages were covered with square focus coils, which provide a top-down electromagnetic field exposure. A transmitted electromagnetic field coil was composed of a printed circuit board with 20 turns of square rolling copper, which has a width of $6 \mathrm{~cm}$ (Figure 1(b)). The focused coil ensured that the electromagnetic field can concentrate the main energy to the stomach region of rats by postural restriction. Moreover, the total bodies of rats were influenced by PEMFs. Space electromagnetic field strength distribution along the diagonal at a perpendicular distance of $1 \mathrm{~cm}$ to the coil was determined by using a Tesla meter (HT108; Hengtong, Shanghai, China). Meanwhile, the electromagnetic field strength distribution along the perpendicular of the focused coil from the center to $5 \mathrm{~cm}$ was measured. The acquired electromagnetic field intensity data were graphically represented (Figures $1(\mathrm{c})$ and $1(\mathrm{~d})$ ). The central point of the electromagnetic fields reached a maximum strength of $34 \pm$ $0.4 \mathrm{mT}$, and the field strength decreased as the horizontal and perpendicular distance from the central coil increased. During the experiment, electromagnetic fields with $15 \mathrm{~Hz}, 40 \%$ duty cycle, were used. According to the International Commission on Non-Ionizing Radiation Protection (ICNIRP) guidelines, the current PEMF had not the negative biological effects on the organism. The rats were housed in transparent resin cages with air bleed, thereby allowing an approximately $1 \mathrm{~cm}$ distance between the coil and rat dorsum. In addition, background electromagnetic fields were measured as $3 \pm 0.3$ $\mu \mathrm{T}$ in the experiment.

2.4. Gastric Juice pH Values and UI. During the HSU healing, on Days 1, 3, 5, and 7 after the model operation, eight rats in each group were slaughtered by using $1.5 \mathrm{ml} \mathrm{kg-15 \%}$ chloral hydrate injection. The abdominal cavity was cut for gastric mobilization down the centerline from the xiphoid process 


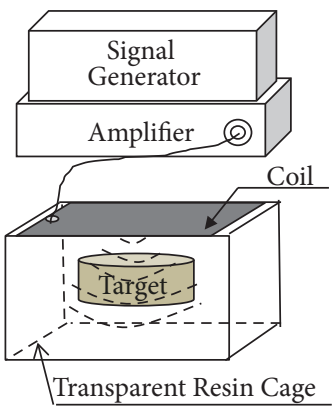

(a)

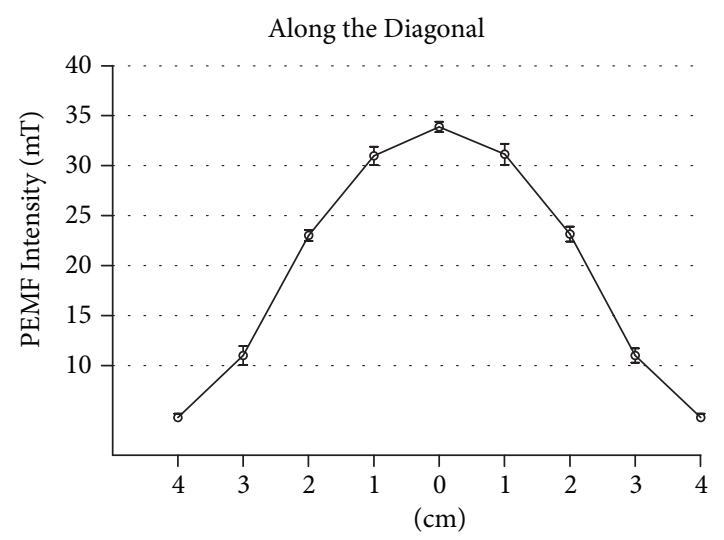

(c)

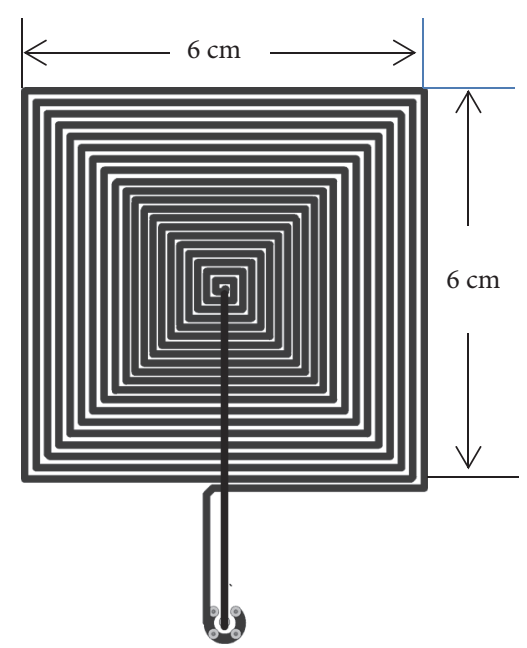

(b)

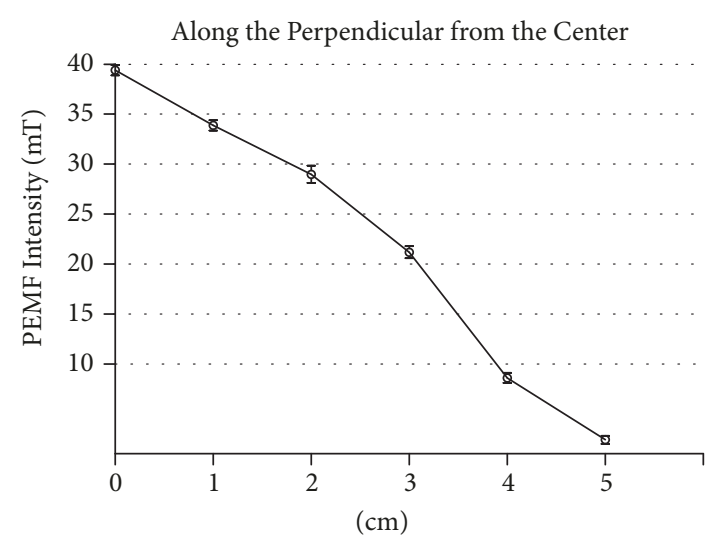

(d)

FIgure 1: (a) Schematic of stimulators used to expose rats to PEMF. (b) Diagram of the focused coil. (c) PEMF spatial distribution along diagonal. (d) PEMF spatial distribution along perpendicular from the center.

to the lower abdomen. After the cardiac and pyloric were nipped with a hemostat, a $\mathrm{pH}$ meter was inserted through a small incision to the stomach to measure the gastric juice $\mathrm{pH}$ values. Then, the stomach was opened along the small curvature and was pinned in the saline with mucosae eversion to conduct macroscopic examination and scoring of UI. Lesion size based on erosion and bleeding conditions was measured along its greatest diameter by using a magnifying glass (Tunçel, Tunçel, \& Aboul-Enein, 2003). Instruction sheets with a five-point scale (1: lesion size $\leq 1 \mathrm{~mm} ; 2: 1 \mathrm{~mm}$ $\leq$ lesion size $\leq 2 \mathrm{~mm} ; 3: 2 \mathrm{~mm} \leq$ lesion size $\leq 3 \mathrm{~mm} ; 4: 3 \mathrm{~mm} \leq$ lesion size $\leq 4 \mathrm{~mm}$; and 5: $4 \mathrm{~mm} \leq$ lesion size) were prepared for the measurement on each group of rats. Five petechial lesions were considered equivalent to a $1 \mathrm{~mm}$ ulcer. The final UI of each group was calculated by dividing the total scores in each group by the number of animals.

2.5. Histopathologic Changes. On Days 1, 3, 5, and 7 after the measurements of $\mathrm{pH}$ and UI values, $2 \mathrm{~mm} \times 5 \mathrm{~mm}$ stomach mucosae samples of eight rats from the HSU and HSU+PEMF groups were harvested for histological examination every time. The ulcer tissue specimens were excised, directly immersed, and fixed in a $10 \%$ formalin solution for $8 \mathrm{~h}$. The samples were embedded in paraffin wax, and $5 \mu \mathrm{m}$-thick sections were cut by using a saw microtome (Leica RM 2155; Leica Instruments, Nussloch, Germany). The sections were deparaffinized, rehydrated, followed by Mayer's hematoxylin and eosin staining. The slides were observed under a light microscope, and the histopathologic differences between the HSU and HSU+PEMF groups were evaluated.

2.6. TNF- $\alpha$ Protein Assay. TNF- $\alpha$ is closely associated with the inflammation indexes of gastric mucosa [18]; thus, TNF$\alpha$ values were detected here. Appropriate amounts of gastric mucosa of eight rats from different groups were cut and homogenized in $400 \mathrm{ml}$ PBS ( $\mathrm{pH}$ 7.2). The homogenates were centrifuged at $3,000 \mathrm{rpm}$ for $10 \mathrm{~min}$ at $4^{\circ} \mathrm{C}$. The supernatant was collected for subsequent analysis. The TNF$\alpha$ concentration was determined by using an enzyme-linked immunosorbent assay kit specific for TNF- $\alpha$ (Biosource, Camarillo, CA, USA) according to the manufacturer's specifications.

2.7. NE Measurements. NE concentrations were measured by using a high-pressure liquid chromatographic (HPLC) assay combined with electrochemical detection (ECD) (Agilent 


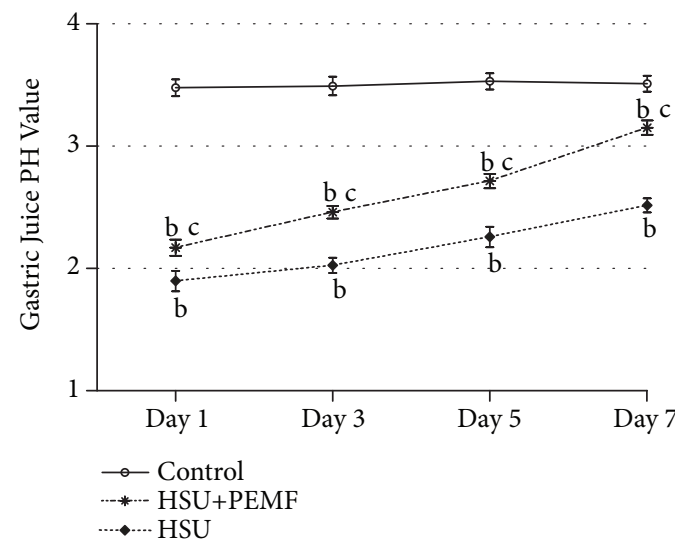

(a)

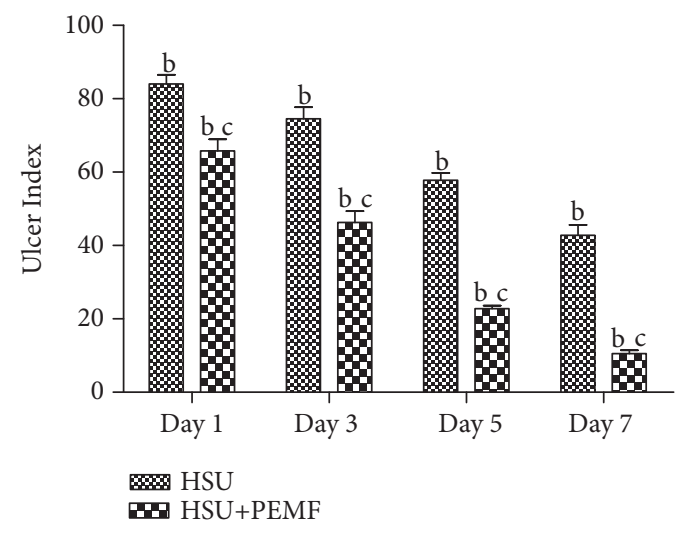

(b)

FIgUre 2: Comparison of the $\mathrm{pH}$ and UI values between the HSU and HSU+PEMF groups on Days 1, 3, 5, and 7. The values are expressed as mean $\pm \mathrm{SD} .{ }^{\mathrm{b}} \mathrm{P}<0.01$ versus the control group and ${ }^{\mathrm{c}} \mathrm{P}<0.05$ versus the HSU group.

100, USA). The hippocampus of rat brains was cryopreserved and smashed with ultrasound. On the basis of an internal standard, $20 \mathrm{ml}$ dihydroxybenzamidine was added to the samples, in which $200 \mu \mathrm{l}(0.1 \mathrm{~mol} / \mathrm{l}) \mathrm{HClO}_{4}$ was added and vortex mixed for $5 \mathrm{~min}$. The resultant homogenates were centrifuged for $10 \mathrm{~min}$. The NE concentrations of the supernatant were measured by HPLC with ECD. During the operation, all the processes were completed by the same person on the basis of the instruction for reagents and instruments.

2.8. Statistical Analysis. All data were expressed as mean \pm deviation (S.D.). Gastric juice $\mathrm{pH}$ values, UIs, histological changes, TNF- $\alpha$ protein, and NE concentrations of each group were analyzed in this study. ANOVA was conducted to evaluate the differences among the various groups. Fisher's least significant difference t-test was applied to determine the significant pairwise differences between the groups. All analyses were performed in SPSS (version 10.0) statistical software for Windows (SPSS, Chicago, IL, USA). Values with $\mathrm{P}<0.05$ were considered statistically significant.

\section{Results and Discussion}

3.1. Gastric Juice pH Values and UIs. Figures 2(a) and 2(b) illustrate the results of average gastric juice $\mathrm{pH}$ values and UIs of different groups on Days 1, 3, 5, and 7. Figure 2(a) demonstrates that the $\mathrm{pH}$ of the control group was similar at approximately $3.49 \pm 0.6$, and the $\mathrm{pH}$ values were lower in the HSU and HSU+PEMF groups than in the control group and increase with time $(\mathrm{P}<0.01)$. However, the $\mathrm{pH}$ values were higher in the HSU+PEMF group than in the HSU group on Days 3, 5, and $7(\mathrm{P}<0.05)$, although no obvious difference was observed on Day 1. In Figure 2(b), the UI difference between the HSU and HSU+PEMF groups was obvious during the ulcer healing. The UI values were higher in the HSU group than in the HSU+PEMF group $(\mathrm{P}<0.05)$. On Day 7 , the UI of the HSU+PEMF group reached $10.25 \pm .03$, thus indicating that the ulcer is nearly restored.
3.2. Histological Changes. Histological examination revealed that no remarkable histological changes occur in the control rats. On Days 1, 3, 5, and 7, the histological changes and differences between the HSU+PEMF and HSU groups were confirmed, as depicted (Figure 3). On Day 1, the normal structure of stomach mucosae nearly disappeared, and considerable edema, inflammatory cell infiltration, and exudative hemorrhage are displayed (Figures 3(a) and 3(e)). However, the degree and incidence of edema, exudative hemorrhage, and inflammatory cell infiltration were remarkably higher in the HSU group than in the HSU+PEMF group. On Days 3 and 5, the structure of stomach mucosae of the two groups accumulated, and the structure was clearer in the HSU+PEMF group than in the HSU group (Figures 3(c) and $3(\mathrm{~g})$ ). During restoration, edema, exudative hemorrhage, and inflammatory cell infiltration remarkably decreased and were still lower in the HSU+PEMF group than in the HSU group. Figures 3(b) and 3(c) exhibit that the newly formed capillaries (red arrows) and fibrous connective tissues of the HSU+PEMF group were obtained, but were not observed in the HSU group (Figures 3(f) and 3(g)). On Day 7, edema, exudative hemorrhage, and inflammatory cell infiltration were nearly absent in the HSU+PEMF group, and the mature capillary network and stomach mucosae were nearly formed (Figure 3(d)). However, sparse inflammatory cells (yellow arrows), hemorrhage (green arrows), and spare edema could still be observed in the HSU group, and some hemorrhage could be found even in deep layer (Figure $3(\mathrm{~h})$ ). In addition, the structure of stomach mucosae was obscure.

3.3. TNF- $\alpha$ Protein. Figure 4 presents that the TNF- $\alpha$ protein of the control group remained at approximately $396 \pm 3 \mathrm{ng} / \mathrm{L}$ on Days 1, 3, 5, and 7. On Days 1, 3, and 5, the TNF- $\alpha$ values were higher in the HSU and HSU+PEMF groups than in the control group $(\mathrm{P}<0.01)$. However, the TNF- $\alpha$ values were significantly lower in the HSU+PEMF group than in the HSU group $(\mathrm{P}<0.05)$. However, on Day 7 , the TNF- $\alpha$ value of the HSU+PEMF group reached approximately $397 \pm 4$, which has no significant difference with the control group $(\mathrm{P}<$ 0.01). Moreover, a remarkable difference between the TNF- $\alpha$ 


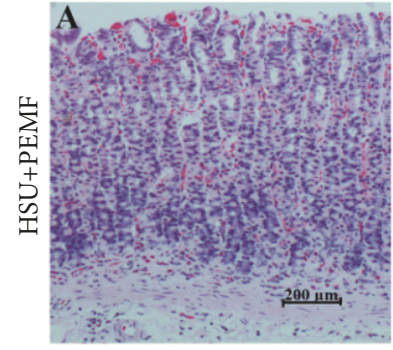

(a)

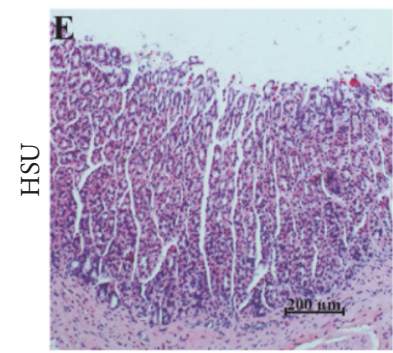

(e)

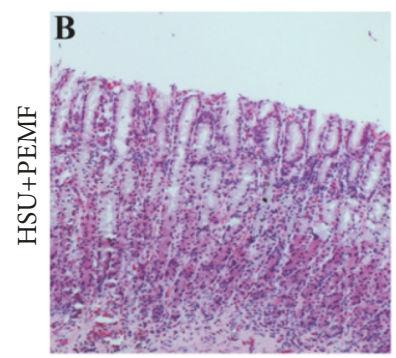

(b)

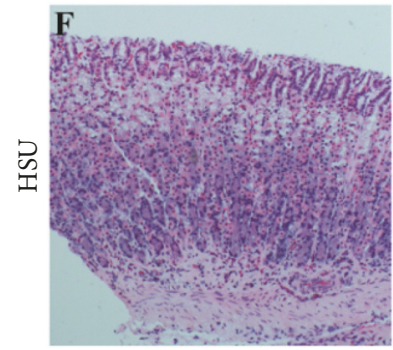

(f)

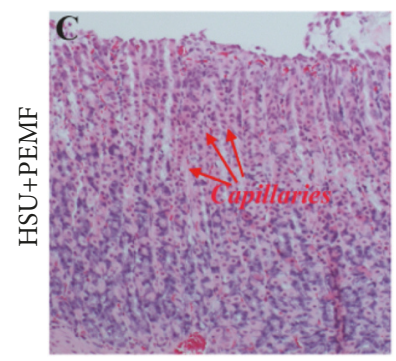

(c)

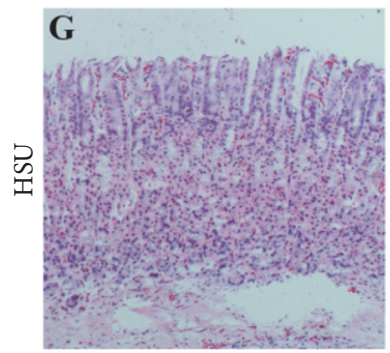

(g)

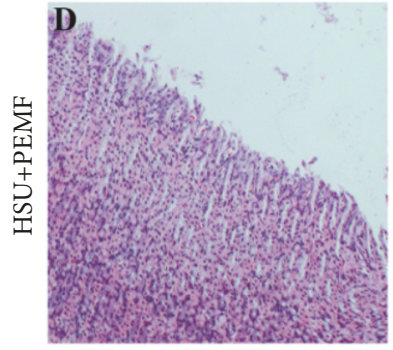

(d)

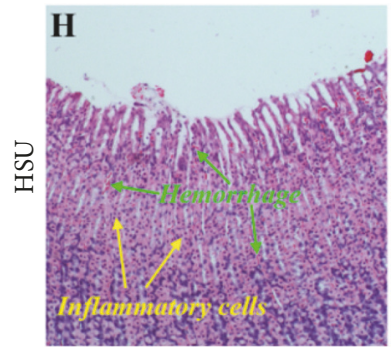

(h)

FIgURE 3: Histology of the HSU+PEMF and HSU wounds in rats (hematoxylin and eosin staining; 10×): (a and e) Day 1, (b and f) Day 3, (c and g) Day 5, and (d and h) Day 7.

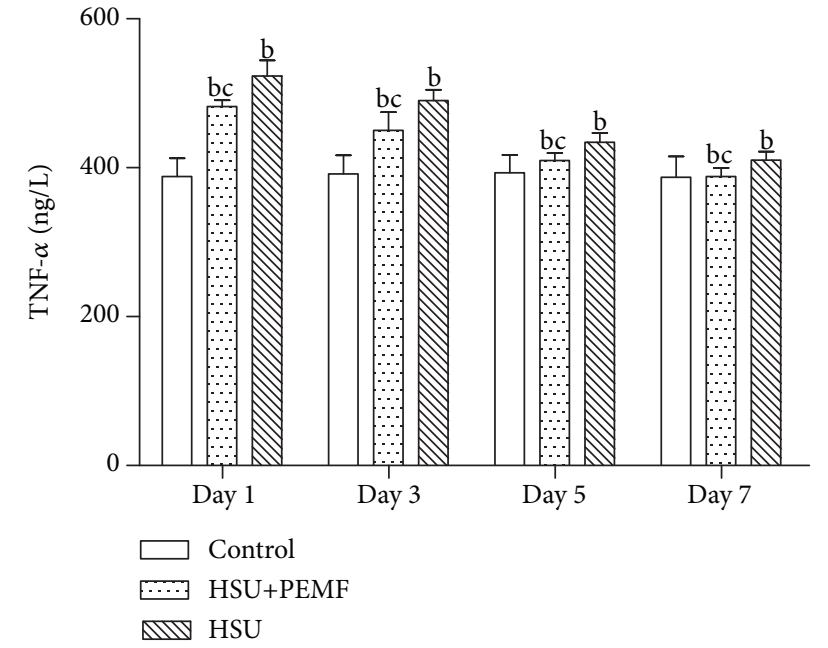

FIgURE 4: Comparison of TNF- $\alpha$ protein between each group on Days $1,3,5$, and 7 . The values are expressed as mean $\pm \mathrm{SD} .{ }^{\mathrm{b}} \mathrm{P}<0.01$ versus control group. ${ }^{\mathrm{c}} \mathrm{P}<0.05$ versus HSU group.

value of the HSU and the control groups was observed. In particular, these results indicated that the inflammation of ulcer of the HSU+PEMF group nearly disappears on Day 7.

3.4. NE. NE is closely related to nervous irritability and can influence stomach acid through the sympathetic nervous system [19]. In Table 1, the NE concentrations of the rats were lower in the control group than in the other groups $(\mathrm{P}<0.01)$ at different time points. The NE concentrations of the HSU and HSU+PEMF groups decreased with time. Moreover, Table 1 summarizes the significant difference between the NE concentrations of the HSU and HSU+PEMF groups from Day
1 to Day 7. In addition, the NE concentrations were lower in the HSU+PEMF group than in the HSU group at different times $(\mathrm{P}<0.05)$. On Day 7 , the NE concentrations of the HSU+PEMF group reached $186.23 \pm 9.29$, which was nearly close to the values of the control group $(\mathrm{P}<0.01)$. However, the NE concentrations remained significantly higher in the HSU group than in the control group.

\section{Discussion}

In the last decades, numerous studies have reported that PEMFs have multiple effects on living organisms by altering the permeability and ion transfer of cellular membranes $[13,20,21]$. Therefore, PEMFs have been investigated for the treatment of a wide spectrum of diseases, such as skin wounds and bone fractures $[16,22]$. However, the effect of low-frequency PEMFs on the wound healing of the inner structure of organisms, such as the stomach, has not been described. In the current study, the effects of low-frequency PEMFs on stomach HSU healing were systematically investigated in HSU rat models. The results indicated that lowfrequency PEMFs can penetrate body tissues to remarkably accelerate HSU healing.

In general, the pathophysiology of stomach HSU is different from normal skin wounds. First, the pathological conditions of the HSU come from physical harm and stress influences $[5,23]$. In addition, the cure of stomach ulcer depends on the anaerobic environment of an abdominal cavity. On the basis of the abovementioned pathophysiological factors and specificity of PEMFs, PEMFs were used to influence the cure of HSU and were verified to improve the cure of HSU. Similar results were reported in our previous studies, in which PEMFs can penetrate tissues to accelerate the healing of frostbite tissues [16]. Moreover, Helicobacter 
TABLE 1: Comparison of NE concentrations (ng/g) between each group on Days 1, 3, 5, and 7.

\begin{tabular}{lcccc}
\hline \multirow{2}{*}{ Group } & \multicolumn{3}{c}{ NE concentrations (ng/g) } \\
& Day 1 & Day 3 & Day 5 & Day 7 \\
\hline Control & $182.81 \pm 11.20$ & $183.32 \pm 4.52$ & $182.19 \pm 9.56$ & $182.33 \pm 6.17$ \\
HSU & $276.25 \pm 7.12^{\mathrm{b}}$ & $268.54 \pm 6.35^{\mathrm{b}}$ & $255.55 \pm 7.36^{\mathrm{b}}$ & $249.36 \pm 4.36^{\mathrm{b}}$ \\
HSU+PEMF & $197.23 \pm 9.21^{\mathrm{b}, \mathrm{c}}$ & $195.71 \pm 7.12^{\mathrm{b}, \mathrm{c}}$ & $192 \pm 6.32^{\mathrm{b}, \mathrm{c}}$ & \\
\hline
\end{tabular}

Values are expressed as mean $\pm \mathrm{SD}$.

${ }^{\mathrm{b}} P<0.01$ versus control group.

${ }^{c} P<0.05$ versus HSU group.

pylori (HP) infection is another factor that can result in HSU [24]. Some works have indicated that the PEMF has bactericidal effect on some bacteria $[25,26]$. However, the effect of PEMF on HP remains unknown. Therefore, the effect of PEMF on HP in vitro and in vivo will be explored as another special subject in our further work. In this study, PEMFs with $15 \mathrm{~Hz}$ have been used because the penetration depth of electromagnetic fields increases with the decrease in frequency. Consequently, all HSUs could be exposed to lowfrequency PEMFs during treatment. In addition, the entire bodies of rats were nearly exposed to PEMFs in the present study although the stomach was located in the central of electromagnetic field energy. The positive effect of the entire exposure style on HSU requires subsequent experiments.

The degree of HSU was closely associated with the $\mathrm{pH}$ values of gastric juice [27]. Our observation revealed that the $\mathrm{pH}$ values of the HSU models were considered below the normal value. Correspondingly, the UI values for the HSU and HSU+PEMF groups were higher on Day 1 than on the other days. The $\mathrm{pH}$ values increased faster in the HSU+PEMF group than in the HSU group with PEMF exposure at different times. Furthermore, the $\mathrm{pH}$ value of the HSU+PEMF group on Day 7 nearly reached the control group. Moreover, the UI values were lower in the HSU+PEMF group than in the HSU group at all time-points. Therefore, PEMFs accelerated the decrease in the UI value, and the increases in $\mathrm{pH}$ values promoted HSU healing. In addition, the HSU group showed that the level of NE is elevated by stress. Similar results were also reported in several previous studies [19, 28]. However, PEMFs effectively decreased the $\mathrm{NE}$ concentrations of rats on the basis of the results of $\mathrm{NE}$ concentrations, which control the stomach neurotransmitter to suppress stomach acids by binding to an adrenergic alpha receptor. Therefore, the series of influence of PEMFs on the HSU group could be called a snowball effect. The influence of PEMFs on NE played a crucial role. However, the effect of PEMFs on the total sympathetic nerve remained unknown. The participation of NE or total sympathetic nerve in the course of HSU healing could not be determined. Therefore, the influence of PEMFs on the total sympathetic nerve should be clarified in subsequent studies.

The histological examination indicated that the ulcer cure is obviously faster in the HSU+PEMF group than in the HSU group. During the examination, the inflammatory cell infiltration diminished quicker in the HSU+PEMF group than in the HSU group, and more mature capillary network and fibrous connective tissue were observed in the HSU+PEMF group than in the HSU group. These results confirmed that PEMFs with proper parameters can promote the healing of injured tissues under hypoxia, especially in the organs, which are nearly similar to our previous research results on the influence of PEMFs on plateau frostbite [16]. However, the effect of PEMFs on inflammatory cells was verified during the detection of TNF- $\alpha$ protein, which is closely associated with inflammation indexes. PEMFs obviously promoted the decrease in TNF- $\alpha$ protein on the basis of the results of TNF- $\alpha$ protein measurement. Therefore, the promotion may accelerate HSU healing. The combined effects of PEMFs were positive during HSU healing.

In this study, the more ideal results of the effects of PEMF on HSU should be obtained in a continuous or periodical hypoxic environment. However, we found that the extremely difficult conditions have made some rats too ill to survive for other complications, such as cerebral edema. Therefore, we checked the effects of PEMF on HSU in the normal environment. When other complications could be controlled, investigating the effects of PEMF on HSU with continuous hypoxic would be truly significant; thus a perfect design should be performed in the future.

\section{Conclusions}

Our study demonstrated that PEMFs can accelerate HSU restoration, which is evidenced by quantitative gastric juice $\mathrm{pH}$ value and UI observation, histological results, TNF$\alpha$ protein, and $\mathrm{NE}$ detection. Based on the results, the findings indicated that PEMF, as an effective noninvasive and accessible therapeutic method, might provide an exciting therapeutic alternative for HSU treatment.

Under some special conditions, PEMF, as an adjuvant therapy, could be combined with drugs to treat gastric ulcer, the actual effects of which require subsequent experiments.

\section{Data Availability}

The detailed data of Figures 2 and 4 used to support the findings of this study are available from the corresponding author upon request. The other data used to support the findings of this study are included within the article.

\section{Conflicts of Interest}

The authors declare that there are no conflicts of interest regarding the publication of this paper. 


\section{Acknowledgments}

We sincerely thank Prof. Hongping Song for their technical support on animal models in our experiments. This work was supported by the China Postdoctoral Science Foundation (Grant No. 2018M633750).

\section{References}

[1] H. Abdelmelek, A. Molnar, S. Servais et al., "Skeletal muscle HSP72 and norepinephrine response to static magnetic field in rat," Journal of Neural Transmission, vol. 113, no. 7, pp. 821-827, 2006.

[2] M. E. Ahmed, B. A. Al-Knawy, A. H. Al-Wabel, and A. K. Foli, "Duodenal ulcer and Helicobacter pylori infection at high altitude: experience from southern Saudi Arabia," Canadian Journal of Gastroenterology \& Hepatology, vol. 11, no. 4, pp. 313316, 1997.

[3] D. Frisancho and O. Frisancho, "Digestive physiology and pathology in high altitude," Revista de Gastroenterología del Perú, vol. 12, no. 3, pp. 155-158, 1992.

[4] B. F. Sisken, M. Kanje, G. Lundborg, and W. Kurtz, "Pulsed electromagnetic fields stimulate nerve regeneration in vitro and in vivo," Restorative Neurology and Neuroscience, vol. 1, no. 3-4, pp. 303-309, 1990.

[5] S. Levenstein, S. Rosenstock, R. K. Jacobsen, and T. Jorgensen, "Psychological stress increases risk for peptic ulcer, regardless of helicobacter pylori infection or use of nonsteroidal antiinflammatory drugs," Clinical Gastroenterology and Hepatology, vol. 13, no. 3, pp. 498-506.e491, 2015.

[6] T.-Y. Wu, S.-Q. Ding, J.-L. Liu et al., "High-altitude gastrointestinal bleeding: An observation in Qinghai-Tibetan railroad construction workers on Mountain Tanggula," World Journal of Gastroenterology, vol. 13, no. 5, pp. 774-780, 2007.

[7] A. N. Barkun, M. Bardou, C. Q. D. Pham, and M. Martel, "Proton pump inhibitors vs. histamine 2 receptor antagonists for stress-related mucosal bleeding prophylaxis in critically ill patients: A meta-analysis," American Journal of Gastroenterology, vol. 107, no. 4, pp. 507-520, 2012.

[8] C.-X. Ji, D.-S. Fan, W. Li et al., "Evaluation of the antiulcerogenic activity of the antidepressants duloxetine, amitriptyline, fluoxetine and mirtazapine in different models of experimental gastric ulcer in rats," European Journal of Pharmacology, vol. 691, no. 1-3, pp. 46-51, 2012.

[9] S. Pongprasobchai, S. Kridkratoke, and C. Nopmaneejumruslers, "Proton pump inhibitors for the prevention of stressrelated mucosal disease in critically-ill patients: A metaanalysis," Journal of the Medical Association of Thailand, vol. 92, no. 5, pp. 632-637, 2009.

[10] D. Bialy, M. Wawrzynska, I. Bil-Lula et al., "Low frequency electromagnetic field conditioning protects against I/R injury and contractile dysfunction in the isolated rat heart," BioMed Research International, vol. 2015, Article ID 396593, 7 pages, 2015.

[11] K. Lim, J. Hexiu, J. Kim et al., "Effects of electromagnetic fields on osteogenesis of human alveolar bone-derived mesenchymal stem cells," BioMed Research International, vol. 2013, Article ID 296019, 2013.

[12] N. Lee, J. Park, Y. Choi, J. Kim, B. Jung, and Y. Lee, "Effect of pulsed electromagnetic fields on the blood circulation in ischemic skin models: A pilot study," Electromagnetic Biology and Medicine, vol. 37, no. 4, pp. 202-207, 2018.
[13] S. Dey, S. Bose, S. Kumar, R. Rathore, R. Mathur, and S. Jain, "Extremely low frequency magnetic field protects injured spinal cord from the microglia- and iron-induced tissue damage," Electromagnetic Biology and Medicine, vol. 36, no. 4, pp. 330340, 2017.

[14] K. Li, L. Gesang, and C. He, "Mechanism of apoptosis involved in gastric mucosal lesions in Tibetans with high-altitude polycythemia," Experimental and Therapeutic Medicine, vol. 14, no. 4, pp. 3780-3787, 2017.

[15] J. A. de Pedro, A. J. Pérez-Caballer, J. Dominguez, F. Collía, J. Blanco, and M. Salvado, "Pulsed electromagnetic fields induce peripheral nerve regeneration and endplate enzymatic changes," Bioelectromagnetics, vol. 26, no. 1, pp. 20-27, 2005.

[16] M. Jiao, L. Lou, L. Jiao et al., "Effects of low-frequency pulsed electromagnetic fields on plateau frostbite healing in rats," Wound Repair and Regeneration, vol. 24, no. 6, pp. 1015-1022, 2016.

[17] Y. Rosli, H. C. Yap, A. Hamid, F. W. Ibrahim, and N. F. Rajab, "Effects of electromagnetic field (EMF) on histological changes and norepinephrine levels in the brains of adult male rats," Jurnal Sains Kesihatan Malaysia, vol. 14, no. 1, pp. 55-61, 2016.

[18] É. Pallagi-Kunstár, K. Farkas, Z. Szepes et al., "Utility of serum TNF- $\alpha$, infliximab trough level, and antibody titers in inflammatory bowel disease," World Journal of Gastroenterology, vol. 20, no. 17, pp. 5031-5035, 2014.

[19] W. P. Pare and A. L Jr, "Brain norepinephrine and stomach ulcers in rats exposed to chronic conflict," Physiology Behavior, vol. 5, no. 2, pp. 217-216, 1970.

[20] M. J. Callaghan, E. I. Chang, N. Seiser et al., "Pulsed electromagnetic fields accelerate normal and diabetic wound healing by increasing endogenous FGF-2 release," Plastic and Reconstructive Surgery, vol. 121, no. 1, pp. 130-141, 2008.

[21] M.-C. Choi, K.-K. Cheung, X. Li, and G. L.-Y. Cheing, "Pulsed electromagnetic field (PEMF) promotes collagen fibre deposition associated with increased myofibroblast population in the early healing phase of diabetic wound," Archives of Dermatological Research, vol. 308, no. 1, pp. 21-29, 2016.

[22] F. Pasi, S. Sanna, A. Paolini et al., "Effects of extremely lowfrequency magnetotherapy on proliferation of human dermal fibroblasts," Electromagnetic Biology and Medicine, vol. 35, no. 4, pp. 343-352, 2016.

[23] M. S. Ali Khan, A. M. Mat Jais, and A. Afreen, "Prostaglandin analogous and antioxidant activity mediated gastroprotective action of Tabernaemontana divaricata (L.) R. Br. flower methanolic extract against chemically induced gastric ulcers in rats," BioMed Research International, vol. 2013, Article ID 185476, 29 pages, 2013.

[24] J. M. Noto, J. Zabaleta, M. B. Piazuelo, P. Correa, and R. M. Peek, "Su1992 role of helicobacter pylori -induced hypoxia-inducible factor-1a in the altitude enigma of gastric carcinogenesis," Gastroenterology, vol. 148, no. 4, pp. 569-570, 2015.

[25] L. Strašák, V. Vetterl, and J. Šmarda, "Effects of low-frequency magnetic fields on bacteria Escherichia coli," Bioelectrochemistry, vol. 55, no. 1-2, pp. 161-164, 2002.

[26] I. Bajpai, N. Saha, and B. Basu, "Moderate intensity static magnetic field has bactericidal effect on E. coli and S. epidermidis on sintered hydroxyapatite," Journal of Biomedical Materials Research Part B: Applied Biomaterials, vol. 100, no. 5, pp. 12061217, 2012.

[27] S. L. Raidal, F. M. Andrews, S. G. Nielsen, and G. Trope, "Pharmacokinetic and pharmacodynamic effects of two omeprazole 
formulations on stomach $\mathrm{pH}$ and gastric ulcer scores," Equine Veterinary Journal, vol. 49, no. 6, pp. 802-809, 2017.

[28] M. F. Nur Azlina and M. I. Nafeeza, "Tocotrienol and $\alpha$ tocopherol reduce corticosterone and noradrenalin levels in rats exposed to restraint stress," Die Pharmazie, vol. 63, no. 12, pp. 890-892, 2008. 


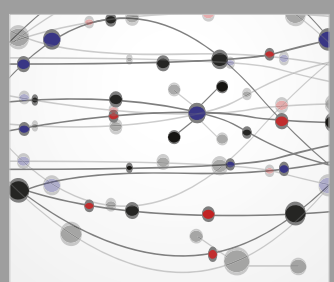

The Scientific World Journal
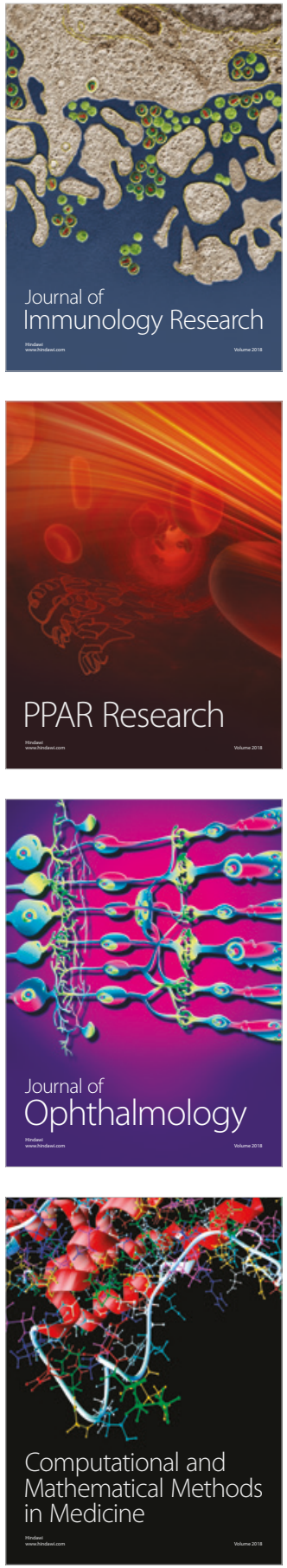

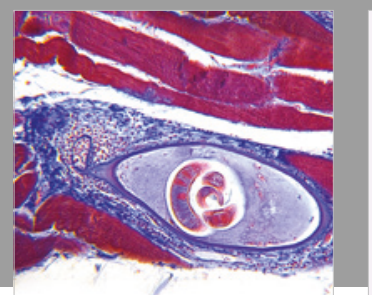

Gastroenterology Research and Practice

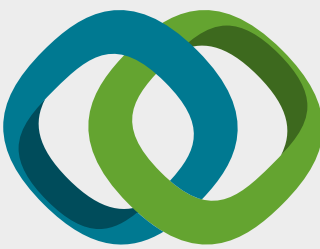

\section{Hindawi}

Submit your manuscripts at

www.hindawi.com
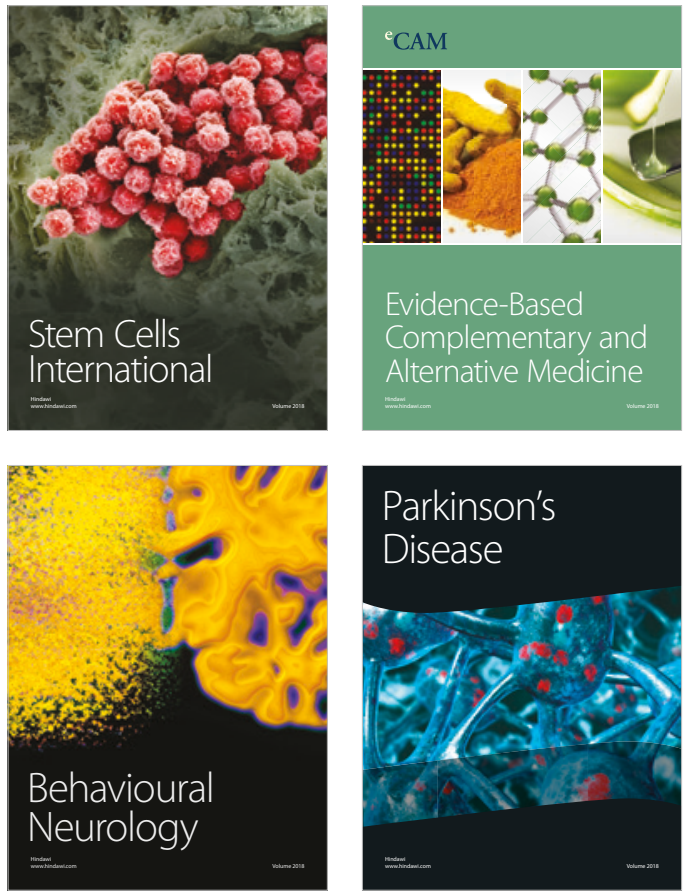

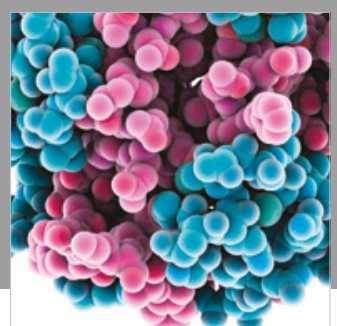

ournal of

Diabetes Research

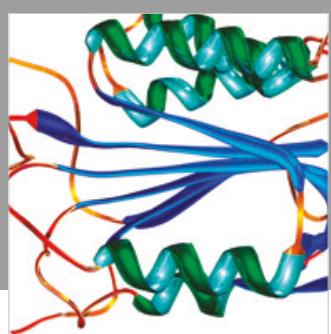

Disease Markers
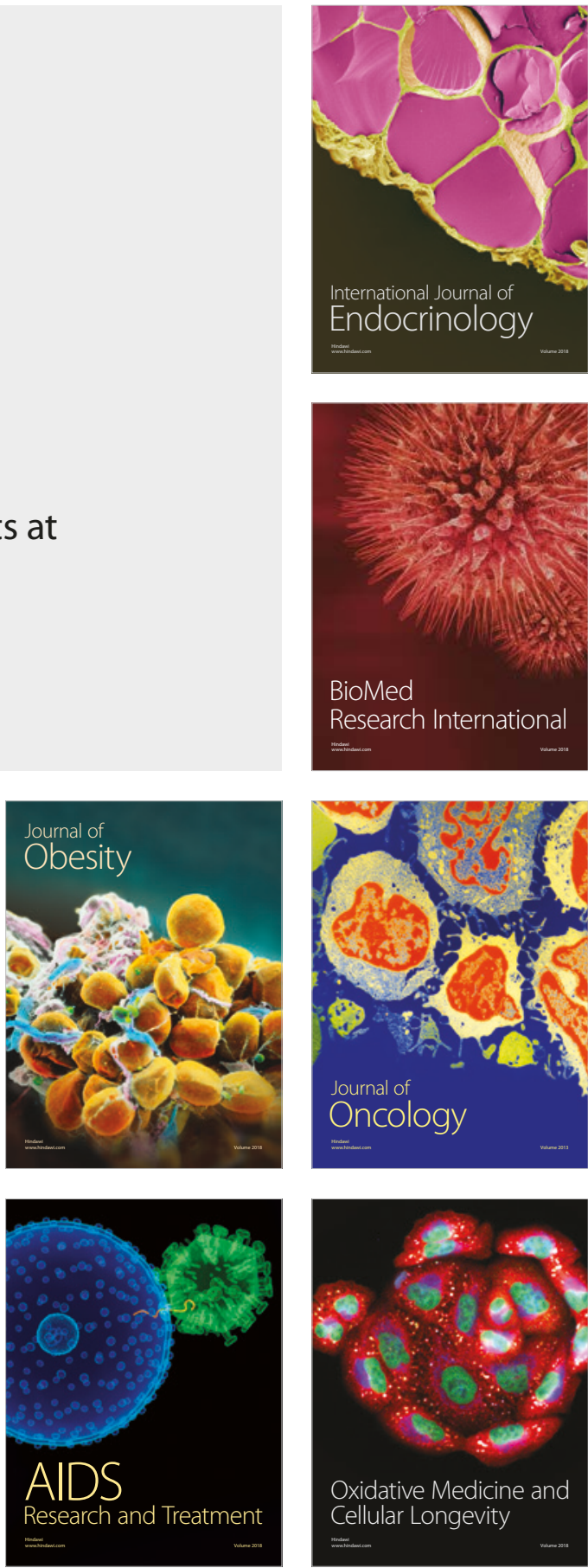